\title{
Needle Insertion in CT Scanner with Image Overlay - Cadaver Studies
}

\author{
Gabor Fichtinger ${ }^{1}$, Anton Deguet ${ }^{1}$, Ken Masamune ${ }^{2}$, Emese Balogh ${ }^{1}$, \\ Gregory Fischer ${ }^{1}$, Herve Mathieu ${ }^{1}$, Russell H. Taylor ${ }^{1}$, Laura M. Fayad ${ }^{1}$, and \\ S. James Zinreich ${ }^{1}$ \\ ${ }^{1}$ Johns Hopkins University, Baltimore, MD, USA \\ ${ }^{2}$ Tokyo Denki University, Japan \\ contact email: gabor@cs. jhu . edu
}

\begin{abstract}
An image overlay system is presented to assist needle placement in conventional CT scanners. The device consists of a flat LCD display and a half mirror and is mounted on the gantry. Looking at the patient through the mirror, the CT image appears to be floating inside the patient with correct size and position, thereby providing the physician with two-dimensional "X-ray vision" to guide needle placement procedures. The physician inserts the needle following the optimal path identified in the CT image that is rendered on the LCD and thereby reflected in the mirror. The system promises to increase needle placement accuracy and also to reduce X-ray dose, patient discomfort, and procedure time by eliminating faulty insertion attempts. We report cadaver experiments in several clinical applications with a clinically applicable device.
\end{abstract}

\section{Introduction}

Numerous studies have demonstrated the potential efficacy of CT-guided percutaneous needle-based therapy and biopsy in a wide variety of medical problems. Contemporary practice, however, is limited by the freehand technique, which requires mental registration of the patient's anatomy to the $\mathrm{CT}$ image in targeting and precise handeye coordination in inserting the needle. This often leads to faulty needle placement attempts followed by repeated CT scans and adjustments, increasing the discomfort and radiation exposure of the patient and lengthening the procedure. Practitioners generally agree that, given enough time and opportunity for intermittent CT imaging and adjustments, the target usually can be reached with appropriate accuracy. The important question is, however, whether the same objective could be achieved with just one attempt, because each correction requires taking an extra CT image (or even series of images) and reinsertion of the needle, which in turn increases the risk of post-procedure complication, discomfort, and radiation exposure to the patient. Therefore, eliminating faulty needle insertion attempts is a prime objective of our research.

A variety of tracked navigation systems and surgical robots have been investigated; all resulting in systems that are prohibitively complex and/or expensive for routine clinical use. Head-mounted displays [1,2], video projections [3], and volumetric image overlay systems [4] have been tried out. Unfortunately, all such methods require 
elaborate calibration, registration, and spatial tracking of all actors and components; a rather complex and expensive engineering entourage. In the pursuit of simpler image overlay systems, our group [5,6] and concurrently Stetten $[7,8]$ have been investigating 2D image reflection produced in a semitransparent mirror. This technique provides an optically stable $2 \mathrm{D}$ reflection image without auxiliary tracking, requiring only a simple pre-operative alignment. As the majority of needle placement procedures are executed "in-plane" (i.e. when the needle is completely contained a single CT slice), giving up 3D rendering for engineering simplicity and low cost appears to be a reasonable tradeoff. This paper describes a clinically deployable embodiment of the Masamune-Stetten concept, optimized for needle placement procedures in conventional $\mathrm{CT}$ scanners. In particular, we report new hardware, display software,

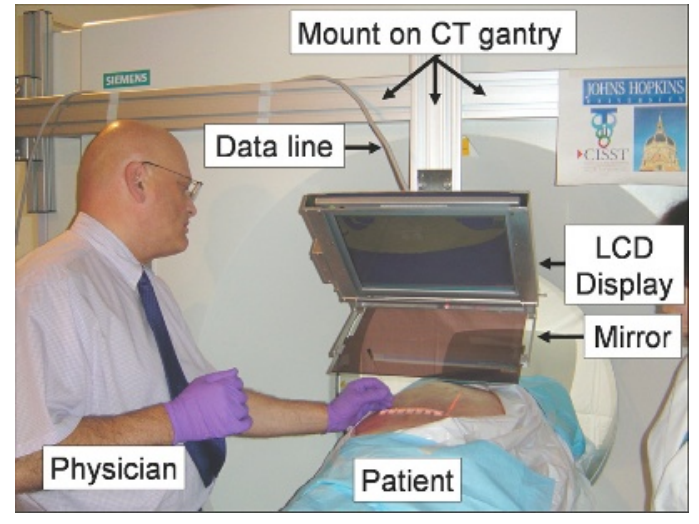

Fig. 1. Spinal needle insertion in human cadaver and the results of phantom and human cadaver studies. Approval is being sought to begin human trials.

\section{System Design}

A flat LCD display and a semi-transparent mirror are mounted on the gantry of a CT scanner (Figure 1) A CT slice is acquired and rendered on the display. The display is positioned with respect to the mirror so that the reflection of the CT image in the mirror coincides with the patient's body behind the mirror (Figure 2), as was described previously in [6]. The overlay is mounted on the gantry, so it tilts with it, if necessary. In many needle placement procedures, after the entry point is selected with the use of skin fiducial markers, three degrees-offreedom (DOF) motion of the needle need to be controlled. The physician uses the overlay image to control the in-plane insertion angle $\left(1^{\text {st }}\right.$ DOF), while holding the needle in the axial plane marked by the gantry's laser light ( $\left.2^{\text {nd }} D O F\right)$. The insertion depth $\left(3^{\text {rd }} \mathrm{DOF}\right)$ is marked on the overlay image. In our proof-of-concept prototype [6], the overlay image was created in the imaging plane of the scanner, which allowed for rapid imaging update, but it prohibitively constrained the workspace. The

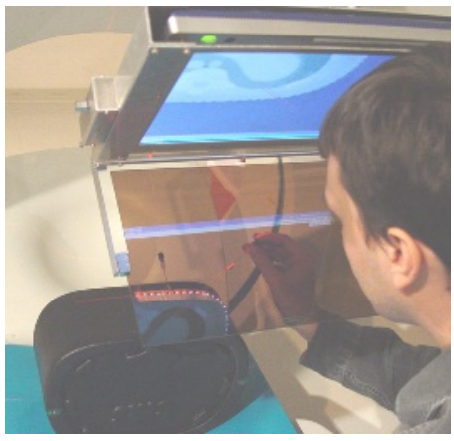

Fig. 2. Needle insertion in phantom. The CT image appears to be floating inside the body with correct alignment and magnification. 
new device uses the outer laser plane of the scanner. While this arrangement provides a conveniently large workspace, it also requires moving the table between imaging and insertion.

The procedural workflow is as follows. The patient is externally marked with IZI CT Biopsy Strip (IZI Corporation, Baltimore, MD). We acquire a CT volume (Siemens Somatom-4), with a slice thickness appropriate for the given application. The plane of insertion is identified and the slice of interest is transferred to the planning and display software running on a WindowsXP laptop. The entry and target points are picked in the CT image, a visual needle guide is displayed, and the scene is pushed to the overlay display as it will be seen later in the left panels of Figure 7. The CT table is translated out by the known offset between the slice of interest and plane of the overlay. The physician places the needle tip at the entry point and lines it up with the visual insertion guide seen in the mirror, while holding the needle in the plane of the laser (Figure 1.) The skin fiducials are visible on both the patient and in the overlay image and coincidence between the corresponding marks indicates correct alignment. This feature is particularly useful when

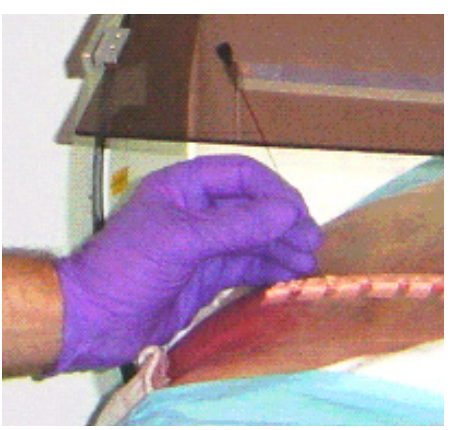

Fig. 3. Needle insertion with single stroke with the assistance of virtual needle guide the target anatomy is prone to motion due to respiration or mechanical force. (This was not found to be a limiting factor in cadaver studies, however.) Usually, a confirmation image is acquired after insertion, as we show later in Figures 6,7, and 8.

\section{Display Software and Registration}

Pre-operative calibration, insertion planning, and display image generation are performed on the stand-alone laptop. The CT image containing the target is transferred from the scanner a DICOM receiver client running on the laptop. The laptop concurrently drives the flat panel LCD display of the overlay device, for which the image is flipped horizontally so that the mirror would flip the image back to normal lateral polarity. The insertion planning component is kept simple and intuitive to speed up the process. We refrain from 3D scenes altogether. In the interactive display, the physician can select a sub-image of interest, for which the magnification, display, and level can be adjusted. The target and entry points are assigned by two mouse-clicks. A visual needle guide and depth-gauge automatically appear on the screen. The computer flips the scene, and after ensuring correct magnification and orientation, pushes the image to the overlay display. The physician holds the needle at the entry point behind the mirror and adjusts the needle to match with the virtual guide. This posture allows the physician to insert the needle with a single stroke along to the marked trajectory, without overdriving (Figure 3.)

After mounting, the display, mirror, and scanner must be aligned. The angle of the display and mirror are fixed with respect to one another and an adjustable arm is used 
to set up the display-mirror unit with respect to the CT laser. For the in-plane registration a phantom made of a $10 \mathrm{~mm}$ thick acrylic board containing an asymmetric set of $5 \mathrm{~mm}$ diameter aluminum pegs is mounted in the overlay plane. A CT image of the pegs is acquired and rendered on the overlay display as seen in Figure 4. The magnification, in-

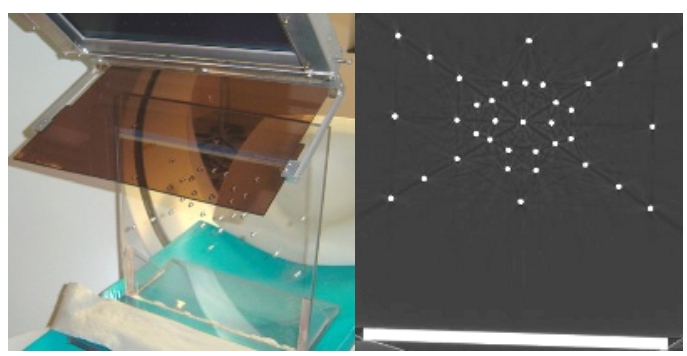

Fig. 4. Calibration phantom and its CT image plane rotation, and translation are adjusted until each aluminum peg coincides with its mark in the CT image.

Calibration has a decisive impact on the system's performance; thus this step must be robust to algorithmic and human errors, while still simple and fast. The marks of the aluminum pegs are auto-segmented in the phantom's CT image and then the technician leans over the mirror and selects the points where the pegs appear in the mirror. From these we calculate the in-plane translation and rotation and then correct the overlay image.

We analyzed the combined effects of human and optical errors on synthetic data, with the objective to determine the optimal number, distribution, and selection order of registration points. We artificially misaligned the two sets of points (i.e. the pegs and their respective image coordinates) by applying a known transformation on each pair and adding noise to each data point, as explained in Figure 5a. Let $Q$ and $R$ be the corresponding points to register in CT and real space. To simulate the error induced by the user and the physical property of the semi-transparent glass, we added some "noise" to the position of $\mathrm{R}$, obtaining point $P$. We registered $P$ to $Q$ getting a rigidbody transformation $T_{\text {reg. }}$. Using this transformation, we transformed $R$ obtaining $S$. Therefore, the system error was the distance between points $S$ and $Q$. We simulated human error by adding a random value ( \pm 2 pixels) to the position of the point to be registered. Parallax error from the mirror may induce a significantly large error. At $d=4 \mathrm{~mm}$ thickness and $\alpha=30^{\circ}$ view angle and using the formulas derived in Figure $5 \mathrm{~b}$, the parallax error was about $\mathrm{x}=1.28 \mathrm{~mm}$, the effect of which fortunately can be substantially reduced by altering the view points during the calibration process. We also concluded that iterative re-registration more favorable than collecting all registration points first and then calculating a single global registration. This observation was true
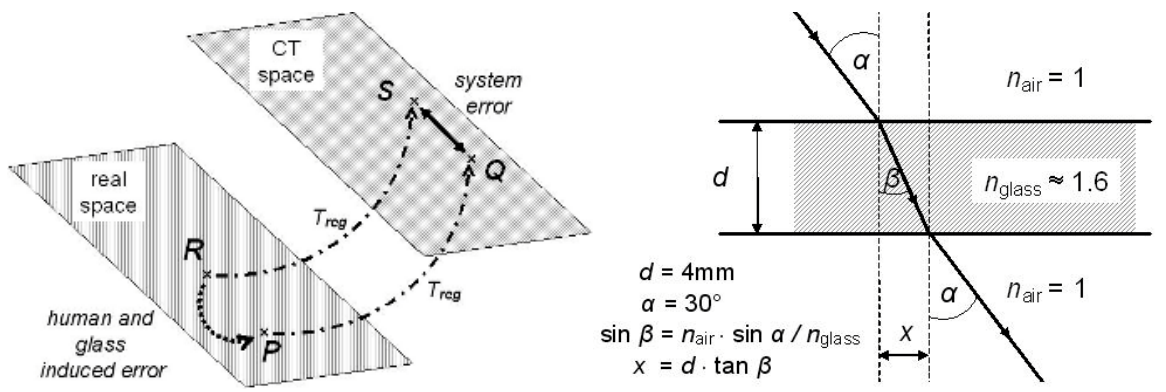

Fig. 5. Definition of registration error (a) and the calculation of parallax error (b) 
across the range of practically relevant amplitudes and distributions of noise and number of registration points. The best strategy was found to be the following: (1) Pick a peg near the area of interest in the vicinity of expected target and entry. Let the computer calculate translation and readjust the overlay image. (2) Pick a peg far out from the previous one. Let the computer calculate rotation and readjust the overlay image. (3) Pick a new peg again relatively close to area of interest to adjust translation. (4) Pick another peg to adjust rotation, far out and in $90^{\circ}$ from the previous one. (5) Repeat the pair-wise process until sufficient registration is observed between the phantom and its CT image. It is particularly important to compensate for the parallax error by altering the viewpoint. The registration error, using four pairs of registration points and assuming \pm 2 pixel human error was $1.23 \mathrm{~mm}(\mathrm{STD}=0.31)$ at $30^{\circ}$ view angle and $0.74 \mathrm{~mm}(\mathrm{STD}=0.21)$ at perpendicular view $\left(\alpha=0^{\circ}\right)$. This performance appears to be sufficiently accurate for most CT-guided needle placement procedures.

\section{Experiments and Results}

Body Phantoms: A male body phantom was fabricated by attaching three cm-thick tissue-equivalent bolus material (Harpell Associates Inc., Oakville, ON, Canada) on the back of a plastic male torso and placing $1.5 \mathrm{~mm}$ diameter metal balls in the bolus at various depths. This phantom represented a male upper body in prone position, with hard fat/muscle layer with mechanical targets in the back, spine, and shoulder. The objectives were to (1) demonstrate accurate needle placement at the pre-marked targets and (2) assess the ergonomics of the system. Experienced and novice interventionalists each executed four needle insertions with $18 \mathrm{G}$ diamond needles. The accuracy of needle placement was assessed in post-insertion $\mathrm{CT}$, with $1 \mathrm{~mm}$ slice thickness. The experienced and novice users alike approached the implanted targets within $2 \mathrm{~mm}$, in every attempt. The device had excellent ergonomics: there was no interference in the workspace and all targets were conveniently accessible. The ergonomics of the system was also tested on a commercial interventional phantom (CIRS, Norfolk, VA) shown in Figure 2, resulting in essentially the same outcome. Also importantly, the reproduction error of the CT gantry mount, without any adjustment, was consistently below $2 \mathrm{~mm}$ (which is less than the width of the laser), tested in 10 trials.

\section{Cadaver Experiments:} The objective of human cadaver experiments was to demonstrate the ability clinically adequate needle placement to various anatomical

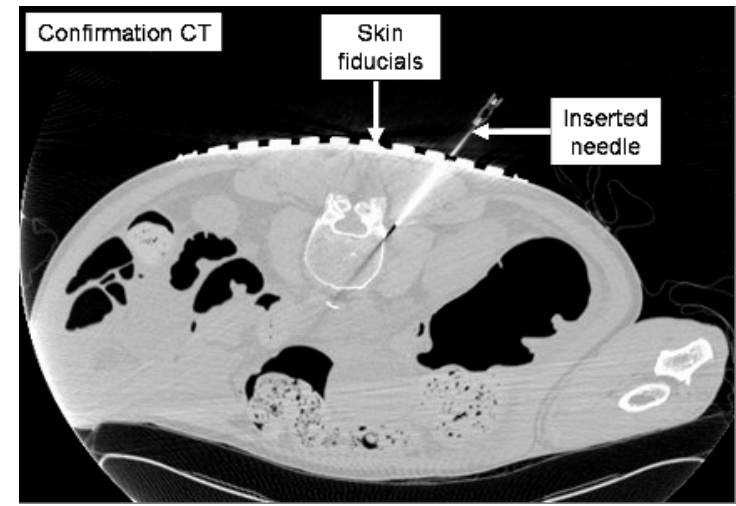

Fig. 6. CT of confirmation of needle placement for spinal pain management 
targets, in several potential clinical applications. The accuracy of needle placement was assessed in post-insertion CT. The slice thickness was 1.0-3.0 mm, which was clinically adequate for the procedures in our study.

Spinal Nerve Blocks and Facet Joint Injections are demanding because the interventional team must perform these bilateral injections under 10 minutes. We positioned the cadaver in prone position for nerve root and facet joint injections in the lumbar spine area in the setup seen in Figure 1. We performed four needle insertions with standard $22 \mathrm{G}$ beveled needles at the L4 and L5. Contrast and therapeutic substances were not injected. All four spinal nerve block and facet joint needle placements produced clinically adequate results, the needle tip landed within $3 \mathrm{~mm}$ from the selected target, without overdriving. One of the confirmation

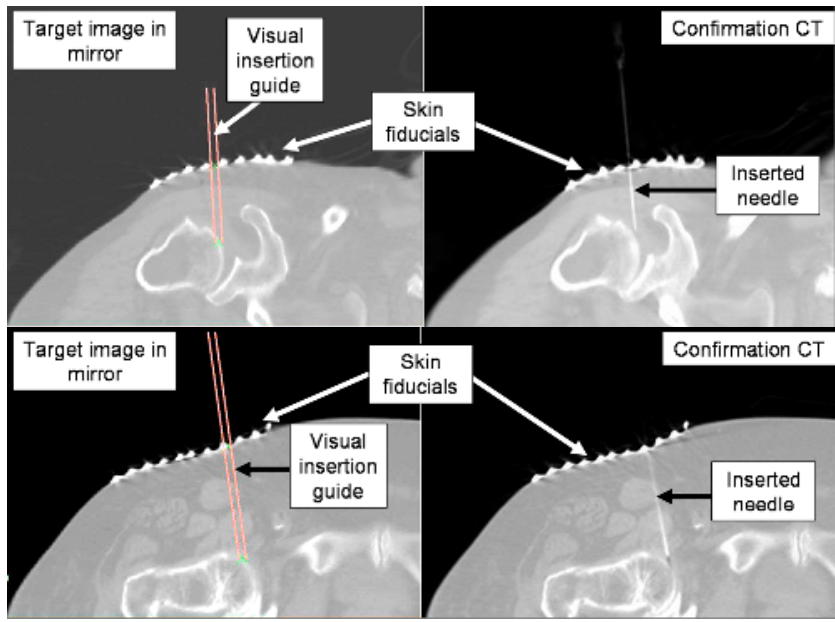

Fig. 7. Target and confirmation CT for arthography of shoulder (top) and hip joint (bottom)

CT images is shown in

Figure 6, where the needle tip touches the correct anatomical target. The needle is completely included in the confirmation image, indicating that the physician managed to keep the needle exactly in the gantry's laser plane.

Shoulder and Hip Arthographies are diagnostic procedures frequently applied for assessment of joint injury. Typically, some MRI contrast agent is injected percutaneously into the injured joint under CT or X-ray fluoroscopy guidance and then the patient is brought to an MRI imaging facility for diagnostic scanning.

We fixed a female cadaver in supine position and performed four needle insertions to the shoulder and hip joint, using standard $22 \mathrm{G}$ beveled needles. The accuracy of needle placement was assessed in intermittent $\mathrm{CT}$ and then contrast substance was also injected in two cases. All four needle placements for joint arthography of the

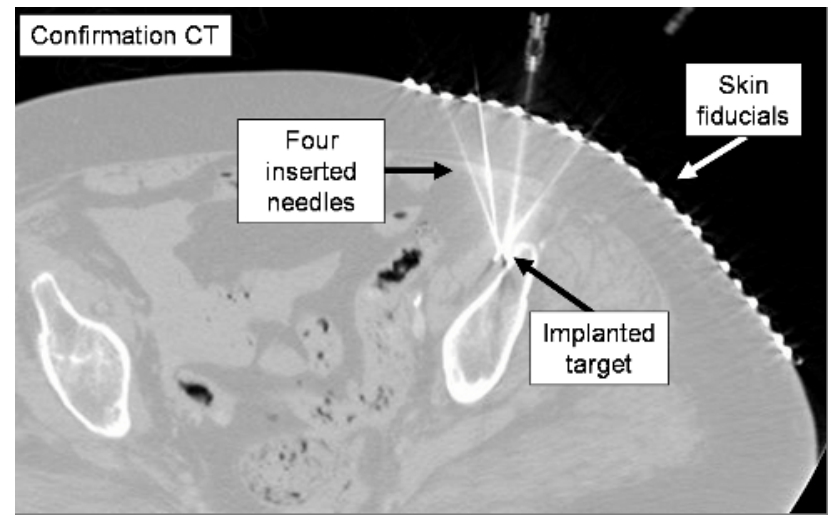

Fig. 8. Confirmation CT of pilot needle insertion in bone biopsy 
shoulder and hip were clinically successful, as the needles landed in the targeted joint space. Two cases are shown in Figure 7. Each needle enters the body in the selected entry point, proceeds along the marked trajectory, and touches the selected anatomical target point in the joint space. Contrast material (not shown in the figures) was very hard to inject into the stiffened joint of the cadaver and it did not distribute as evenly as in live patients.

Pilot Needle for Bone Biopsy: Bone biopsies are demanding procedures because the needle must make contact with the bone in some predefined angle that is often very difficult to achieve freehand. One must also avoid sensitive structures (like nerves and blood vessels) in the path. As we almost always operate on damaged bone, faulty placement and misalignment of the large needle or drill may cause structural damage to the already weakened bone; in fact, fracture is not an insignificant intraoperative risk. Many patients have preexisting micro-fractures, a condition that may develop into a full fracture if the instrument is accidentally wedged in the fracture line. Altogether, unassisted freehand planning of the skin entry, trajectory, bone entry, and cortical target can become exceedingly difficult.

We fixed a female cadaver in supine position and performed four needle insertions to the pelvis, using standard $22 \mathrm{G}$ beveled needles. A $1.5 \mathrm{~mm}$ metal ball marker was implanted percutaneously into the right lateral wall of the left pelvis, and then this location was targeted from several directions from different entry points. All four the pilot needle insertions for pelvic bone biopsy were clinically successful. A confirmation CT is shown in Figure 8. Unfortunately, the artifact caused by the needles suppresses the signal of the implanted metal target, but the needles converge in a confined area, indicating adequate accuracy for bone biopsy. Three of the four needles are completely included in the image slice, while the fourth one is slightly deflected from the image plane.

\section{Discussion}

In our previous prototype the image overlay device was mounted in the inner laser plane of the scanner, the physician's workspace was often restricted and several targets were not accessible [6]. Therefore, the unanimous decision of the participating physicians was to use the outer laser plane of the scanner, even though it required translation of the CT table between imaging and needle insertion. Needle insertion was more accurate in phantoms than in human cadavers, for several reasons. First, the phantom tissue was softer and more homogeneous than human cadavers. Also, in phantoms we used $18 \mathrm{G}$ diamond tip needles that barely deflected during insertion, while in the human cadavers we used $22 \mathrm{G}$ beveled needles that had a tendency to deflect in the hard and inhomogeneous tissues. Less experienced users had difficulty in holding the needle in the plane of the laser. Although this presently does not seem to be a clinically significant impediment, we consider applying some form of mechanical constraint to aid the physician with holding the needle in the laser plane.

Although respiration could not be simulated in the passive phantoms and cadavers, the IZI Biopsy Strip fiducials placed on the patient around the insertion point are expected to provide robust real-time indication of any patient motion, in which case 
the insertion can be halted till the patient's body is correctly re-registered. Alternatively, the fiducials can be used for re-registration of the patient, in the same manner as the calibration fixture is registered.

We have encountered several minor issues that could prove to be significant as our efforts are gearing up toward clinical trials. For example, it is inconvenient to have the planning laptop in the scanner room and its wire connection may also disrupt normal traffic. (In the clinical device, the laptop will be situated next to the console and apply wireless communication to the mouse used for calibration. Also, slight changes in backlight and glare on the display frame have also caused difficulties.)

Adequate needle placement was confirmed in post-insertion CT imaging in all insertion attempts, without needing re-insertion and intermittent CT. The workflow and instrumentation have not been optimized for time and the ergonomics of the system still need to improve. Nevertheless, the system was found to be adequate for initial human trials in several needle placement applications and the cadaver studies were predicative of good clinical performance. Presently, IRB approval is being sought to commence human trials while software and hardware refinements will continue.

Acknowledgements. Funding was available from NSF EEC-9731478, Siemens Corporate Research (Princeton, NJ), and Japanese Ministry of Education, Science, Sports and Culture, Grant-in-Aid for Young Scientists \#14702071. We are grateful to Beatrice Mudge, RTT for her help throughout the experiments and Frank Sauer, PhD (Siemens) for his advice.

\section{References}

1. Sauer F, Khamene A, Vogt S: An Augmented Reality Navigation System with a SingleCamera Tracker: System Design and Needle Biopsy Phantom Trial. Fifth International Conference on Medical Image Computing and Computer-Assisted Intervention, Lecture Notes in Computer Science 2489, pp 116-124, Springer, 2002.

2. Birkfellner, W. Figl, M. Huber, K. Watzinger, F. Wanschitz, F. Hummel, J. Hanel, R. Greimel, W. Homolka, P. Ewers, R. Bergmann, H: A head-mounted operating binocular for augmented reality visualization in medicine - design and initial evaluation, IEEE Transactions on Medical Imaging, Volume: 21, No. 8, pp 991-7, 2002.

3. Grimson WEL, Lozano-Perez L, Wells WM, Ettinger GJ, White SJ, Kikinis R: An Automatic Registration Method for Frameless Stereotaxy, Image Guided Surgery, and Enhanced Reality Visualization, IEEE Trans. Med. Imag. Vol.15, No.2, 1996.

4. Blackwell M, Nikou C, DiGioia AM, Kanade T: An Image Overlay System for Medical Data Visualization, First International Conference on Medical Image Computing and Computer-Assisted Intervention, Lecture Notes in Computer Science 1496, pp.232-240, Springer, 1998.

5. Masamune K, Masutani Y, Nakajima S, Sakuma I, Dohi T, Iseki H, Takakura K: Threedimensional Slice Image Overlay System with Accurate Depth Perception for Surgery, Third International Conference on Medical Image Computing and Computer-Assisted Intervention, Lecture Notes in Computer Science 1935, pp.395-402, Springer, 2000.

6. Masamune K, Fichtinger G, Deguet A, Matsuka D, Taylor RH: An Image Overlay System with Enhanced Reality for Percutaneous Therapy Performed Inside CT Scanner, Fifth International Conference on Medical Image Computing and Computer-Assisted Intervention, Lecture Notes in Computer Science 2488, Part 2, pp 77-84, Springer, 2002. 
7. Stetten G, Chib V: Magnified Real-Time Tomographic Reflection, Fourth International Conference on Medical Image Computing and Computer-Assisted Intervention, Lecture Notes in Computer Science 2208, pp.683-690, Springer, 2001.

8. Stetten GD, Cois A, Chang W, Shelton D, Tamburo RJ, Castellucci J, von Ramm O: CMode Real Time Tomographic Reflection for a Matrix Array Ultrasound Sonic Flashlight. Sixth International Conference on Medical Image Computing and Computer-Assisted Intervention, Lecture Notes in Computer Science 2879, pp 336-343, Springer, 2003. 